\title{
Shape-Based, Low-Thrust Trajectory Design in Alternating Rotational Coordinate System*
}

\author{
Jun MATSUMOTO ${ }^{\dagger}$ and Junichiro KAWAGUCHI
}

Institute of Space and Astronautical Science, Japan Aerospace Exploration Agency, Sagamihara, Kanagawa 252-5210, Japan

\begin{abstract}
This paper proposes a new strategy to design low-thrust trajectories using a shape-based method and an alternating rotational coordinate system. The shape-based method is a well-known strategy for designing spiral trajectories. In this method, a shape of the trajectory is first given and thrust profiles are then derived so that the equations of motion are satisfied. The alternating rotational coordinate system introduced in this paper is a rotational coordinate system in which the angular velocity varies depending on the position of the spacecraft. The advantage of this coordinate system is that spiral trajectories can be described with a simple shape. By combining these two tools, the given shape in the shape-based method becomes simple. In this paper, Sun-Earth L2 to Sun-Mars L1 transfer trajectories are designed as a demonstration of this strategy.
\end{abstract}

Key Words: Spiral Trajectory, Shape-Based Method, Alternating Rotational Coordinate System

\section{Nomenclature}

$A$ : shape function parameter

$a_{r}$ : radial acceleration of the spacecraft

$a_{\theta}$ : tangential acceleration of the spacecraft

$a_{0}$ : magnitude of constant acceleration

$B$ : shape function parameter

$C$ : shape function parameter

$D$ : shape function parameter

$f(r)$ : function of $r$

$G_{r}$ : radial gravitational perturbation

$G_{\theta}$ : tangential gravitational perturbation

$r$ : radial position of the spacecraft

$r_{\mathrm{L} 1}$ : radius of $\mathrm{L} 1$ point

$r_{\mathrm{L} 2}$ : radius of $\mathrm{L} 2$ point

$r_{1}$ : radius of initial circular orbit

$r_{2}$ : radius of terminal circular orbit

$t_{1}$ : parameter of bisection method

$t_{2}$ : parameter of bisection method

$t_{c}$ : parameter of bisection method

$\Delta V:$ change of velocity

$\theta$ : angle between the spacecraft

$\theta_{1}$ : parameter of bisection method

$\theta_{2}$ : parameter of bisection method

$\theta_{c}$ : parameter of bisection method

$\theta_{\mathrm{S} / \mathrm{C}}:$ terminal phase of the spacecraft

$\theta_{\text {planet }}$ : terminal phase of the target planet

$\mu$ : gravitational constant

$\phi(r)$ : function of $r$

$\omega$ : angular velocity

(C) 2018 The Japan Society for Aeronautical and Space Sciences *Presented at the 29th International Symposium on Space Technology and Science, 2-9 June 2013, Nagoya, Japan.

Received 25 April 2017; final revision received 19 March 2018; accepted for publication 11 April 2018.

†Corresponding author, matsumoto.jun@isas.jaxa.jp

\section{Introduction}

In recent years, the use of electric propulsion systems has become a trend for deep-space missions because of the high specific impulse. Deep Space 1, ${ }^{1)}$ Hayabusa, ${ }^{2)}$ SMART-1, ${ }^{3)}$ Dawn, ${ }^{4)}$ Hayabusa2 ${ }^{5)}$ and Procyon, ${ }^{6)}$ which are actual deep-space missions, have utilized ion engines as the main engine in deep space.

Designing strategies for the continuous low-thrust trajectory has been well studied. ${ }^{7,8)}$ The problem is summarized as finding a trajectory that satisfies restrictions, such as boundary conditions. In order to solve this problem, several strategies have been proposed such as (1) the Analytical Approach and (2) the Numerical Approach.

(1) The Analytical Approach is a straight-forward way to solve the problem. In this approach, use of the optimal control theory ${ }^{9)}$ or shape-based method ${ }^{10,11)}$ has been proposed. The optimal control theory is a general way to solve boundary value and optimization problems; however, it is difficult to derive analytical solutions, especially in a highly non-linear problem such as spacecraft trajectory design. The shapebased method first assumes the shape of a low-thrust trajectory in a functional form. In this way, the optimal trajectory cannot be determined; however, the sub-optimal trajectory derived can be used as the initial guess for the numerical simulations. Petropoulos and Longuski ${ }^{10)}$ and Wall and Conway ${ }^{11)}$ have used an exponential sinusoid as a shape function as it expresses the shape of the spiral trajectory.

(2) The Numerical Approach is a trajectory design strategy that uses a numerical optimizer such as a co-location method $^{12)}$ or genetic algorithm. ${ }^{13)}$ Since the advancement in computer power, it has become possible to perform this kind of calculation with ease. However, the initial guess including discontinuous events, such as planet fly-by and impulsive $\Delta V$, needs to be determined before the calculation starts. 
In this paper, (1) The Analytical Approach, a shape-based method is applied to the design of low-thrust trajectories. A unique point of the study is the use of a new rotational coordinate system called the Alternating Rotational Coordinate System (ARCS). The ARCS has a variable angular velocity that is determined depending on the position of the spacecraft so that the spiral trajectory can be expressed simply. The use of the ARCS contributes to expanding the capability of the shape-based method.

This paper first introduces the ARCS and the shape-based method. Secondly several low-thrust trajectories are designed as an example using two- and four-body problems.

\section{ARCS and the Shape-Based Method}

\subsection{ARCS}

\subsubsection{Definition}

The ARCS is a polar rotation system defined in plane. Its angular velocity, $\omega$, depends on the position of the spacecraft and it is defined by the following equations:

$$
\begin{aligned}
\omega(r) & =\sqrt{\frac{\mu}{r^{* 3}}}, \\
r^{*} & =f(r) .
\end{aligned}
$$

Figure 1 summarizes the definition of the ARCS.

\subsubsection{Equations of motion}

In the ARCS, the equations of motion of the spacecraft can be written as

$$
\begin{gathered}
\ddot{r}-r \dot{\theta}^{2}-2 \omega r \dot{\theta}-\omega^{2} r=-\frac{\mu}{r^{2}}+G_{r}+a_{r}, \\
r \ddot{\theta}+2 \dot{r} \dot{\theta}+2 \omega \dot{r}+\dot{\omega} r=G_{\theta}+a_{\theta} .
\end{gathered}
$$

In these equations, the mass consumption of the spacecraft is neglected.

\subsubsection{Demonstration}

As an example, the classical Hohmann transfer trajectory from Earth to Mars is described in the ARCS. In this demonstration, the angular velocity of the ARCS is defined by the following equations:

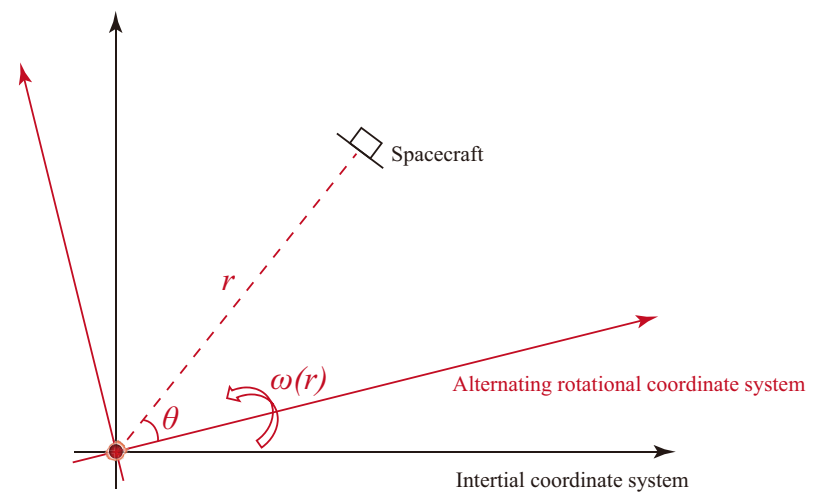

Fig. 1. Definition of ARCS.

$$
\begin{aligned}
\omega(r) & =\sqrt{\frac{\mu}{r^{* 3}}}, \\
r^{*} & =f(r)=r .
\end{aligned}
$$

The angular velocity of the ARCS and the angular velocity of the circular motion in the two-body problems are identical. ${ }^{14)}$ This means that the ARCS roughly follows the motion of the spacecraft. Figure 2 shows the Hohmann transfer in the Inertial Coordinate System (ICS) and the ARCS, respectively. In the ARCS, the trajectory (green line in Fig. 2) can be described in a narrow area due to the ARCS rotation.

\subsection{Shape-based method}

In this study, the shape-based method is applied to the trajectory design. In general, the shape of the trajectory and profiles of the continuous thrust are calculated at the same time by the optimizers. In the shape-based method, however, these two can be considered separately. The strategy of this method is as follows:

Step 1 The shape of the trajectory is first defined in the functional form named "shape function."

Step 2 Thrust profiles are derived by substituting the shape function into the equations of motion.

The shape-based method assumes that the motion of the spacecraft is restricted to a one-dimensional curve. Therefore, the degrees of freedom of the spacecraft motion can be reduced. In this paper, the shape function is defined as:

$$
\theta=\phi(r) \text {. }
$$
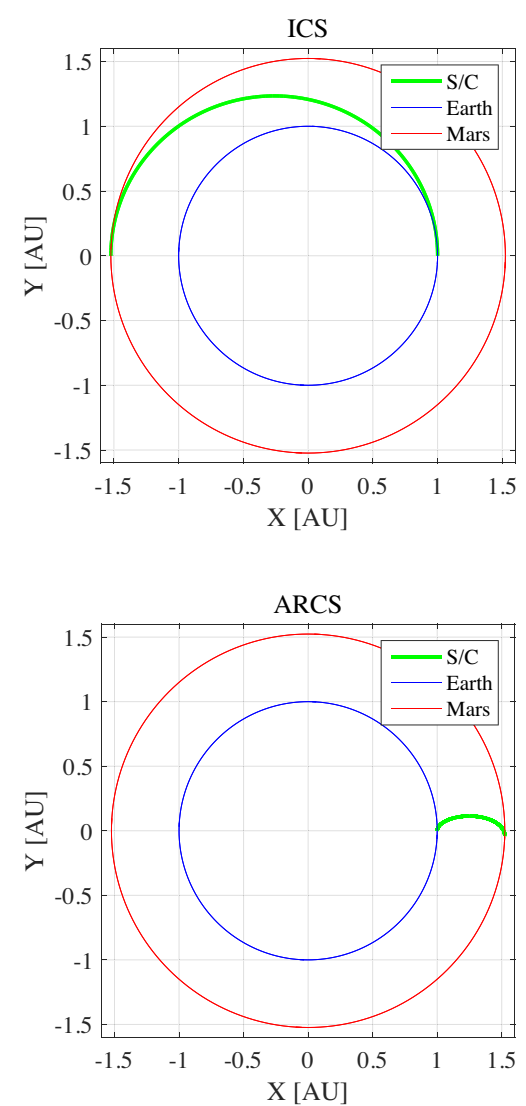

Fig. 2. Hohmann trajectory (upper: ICS, lower: ARCS). 
By differentiating this shape function, the following equations are derived:

$$
\begin{aligned}
& \dot{\theta}=\dot{r} \frac{d \phi}{d r}, \\
& \ddot{\theta}=\dot{r}^{2} \frac{d^{2} \phi}{d r^{2}}+\ddot{r} \frac{d \phi}{d r} .
\end{aligned}
$$

By substituting Eqs. (8) and (9) into Eqs. (3) and (4), one constraint for the thrust profiles is derived as:

$$
a_{\theta}+p a_{r}=q
$$

where,

$$
\begin{aligned}
p= & -r \frac{d \phi}{d r} \\
q= & \left(r \dot{r}^{2} \frac{d^{2} \phi}{d r^{2}}+2 \dot{r} \dot{\theta}+2 \omega \dot{r}+\dot{\omega} r-G_{\theta}\right) \\
& +r \frac{d \phi}{d r}\left(r \dot{\theta}^{2}+2 \omega r \dot{\theta}+\omega^{2} r-\frac{\mu}{r^{2}}+G_{r}\right) .
\end{aligned}
$$

In order to determine the thrust profiles completely, one more constraint needs to be defined.

Constraint (1): Constant thrust condition The simplest constraint is that the magnitude of the thrust acceleration keeps constant, as follow:

$$
a_{r}^{2}+a_{\theta}^{2}=a_{0}^{2}
$$

where, $a_{0}$ is the magnitude of constant acceleration induced by the electric propulsion system. By simplifying Eqs. (10) and (13), thrust profiles can be analytically derived as:

$$
\begin{aligned}
& a_{r}=\frac{p q \pm \sqrt{\left(1+p^{2}\right) a_{0}^{2}-q^{2}}}{1+p^{2}}, \\
& a_{\theta}=\frac{q \mp p \sqrt{\left(1+p^{2}\right) a_{0}^{2}-q^{2}}}{1+p^{2}} .
\end{aligned}
$$

This calculation is graphically shown in Fig. 3 (left). Point A1 in Fig. 3 corresponds to the sign + in Eq. (14) and in Eq. (15). Point A2 corresponds to the sign - in Eq. (14) and + in Eq. (15). That is, there is one degree of freedom to select the sign mathematically. This freedom physically corresponds to having a choice of the direction in which the spacecraft moves. These analytical solutions are discarded when the number in the square root becomes negative. From a mathematical point of view, there are no points where the line and the circle intersect in Fig. 3 (right). Physically, this means that it is impossible for the spacecraft to move along the trajectory using constant thrust.

Constraint (2): Minimized thrust condition Another constraint is that the magnitude of the acceleration induced by the electric propulsion system is minimized. Using Eq. (10), the square of the thrust acceleration magnitude is written as:

$$
a_{r}^{2}+a_{\theta}^{2}=\left(1+p^{2}\right)\left(a_{r}-\frac{p q}{1+p^{2}}\right)^{2}+\frac{q^{2}}{1+p^{2}} .
$$

In order to minimize this magnitude, the thrust profiles need
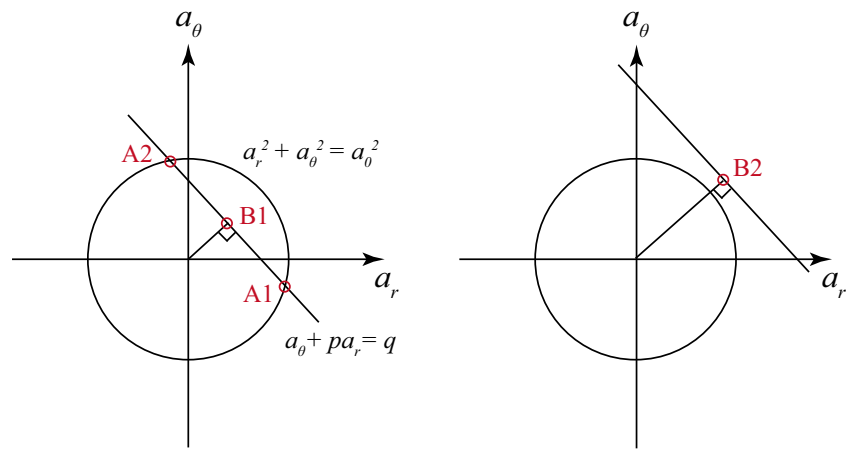

Fig. 3. Thrust profiles determination process.

to be:

$$
\begin{aligned}
& a_{r}=\frac{p q}{1+p^{2}}, \\
& a_{\theta}=\frac{q}{1+p^{2}} .
\end{aligned}
$$

This calculation is graphically shown in Fig. 3. Points B1 and B2 in Fig. 3 correspond to these analytical solutions. Different from the constant thrust condition, these solutions have no square roots. Therefore, calculation failure does not occur.

\subsection{Strategy of trajectory design}

Assumption In this paper, planet-to-planet circular-tocircular transfer is considered. In this case, boundary conditions are selected such that the relative velocity of the spacecraft in respect to the planet is equal to zero.

Determination of ARCS angular velocity Before designing the trajectories, the angular velocity of the ARCS needs to be determined. In order to simplify the boundary conditions, the definition of the angular velocity is restricted as follows:

$$
\omega\left(r=r_{\text {planet }}\right)=\omega_{\text {planet }} .
$$

This means that the angular velocity of the ARCS and planet should be identical at the boundary. By this definition, the boundary conditions can be written such that the velocity of the spacecraft in the ARCS is zero at the planet. According to Eq. (8), $\dot{\theta}$ becomes zero when $\dot{r}$ is zero in the ARCS. Therefore, the boundary conditions can be rewritten to impose $\dot{r}$ equal to zero at the planet. This simplification of the boundary conditions is an important feature of the ARCS.

Boundary value problem solver In order to satisfy the boundary conditions, the sign of the thrust direction in Eqs. (14) and (15) is changed as shown in Fig. 4 (left), which assumes $r_{1}<r_{2}$. In a former part of the trajectory, the sign in Eq. (14) is selected as + to accelerate. In a latter part of the trajectory, the sign in Eq. (14) is selected as - to decelerate so that $\dot{r}$ becomes zero at the terminal position. If we use trajectories toward the inner region of the system $\left(r_{1}>r_{2}\right)$, the sign selection is conducted in reverse. The switching point is determined through a convergent calculation, such as a bisection method.

In Constraint (2), Eqs. (17) and (18), a sign cannot be 

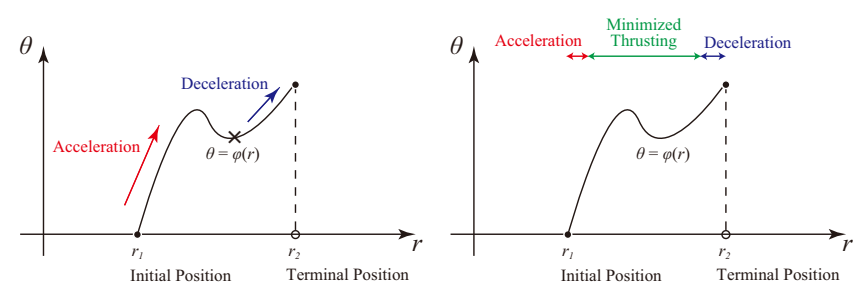

Fig. 4. Trajectory designing procedure.

chosen. Therefore, Eqs. (17) and (18) cannot be used as they are since the boundary conditions cannot be satisfied. In this paper, assistance is applied by using Eqs. (14) and (15), as shown in Fig. 4 (right). Thus, the constant thrust condition is applied in order to satisfy boundary conditions in the first and last of the trajectory.

Algorithm The algorithm of the spacecraft trajectory design is summarized as follows:

Step 1 Defining the angular velocity of the ARCS.

Step 2 Defining the shape function.

Step 3 Determining the switching point through the bisection method.

Step 4 (if needed) Determining the initial phase of the target planet such that the spacecraft has a rendezvous with the planet using the bisection method.

Step 5 Re-calculating the trajectory based on the results of Steps 3 and 4.

Figure 5 shows the flowchart of this algorithm. In Constraint (2), the acceleration duration needs to be given before the calculation (deceleration duration is automatically calculated using the bisection method).

\section{Two-Body Problems}

In this section, a simple trajectory from Earth to Mars transfer in two-body problems is designed as a demonstration. Note that the phase of the planet is not considered here.

\subsection{Formulation}

Definition of the ARCS In this problem, the angular velocity of the ARCS is defined as in Eqs. (5) and (6).

Definition of shape function One possible, and the simplest, shape function is the constant function defined as follows:

$$
\theta=\phi_{s}(r)=\text { const. }
$$

By differentiating this shape function, the following equations are derived:

$$
\begin{aligned}
\frac{d \phi_{s}}{d r} & =0, \\
\frac{d^{2} \phi_{s}}{d r^{2}} & =0 .
\end{aligned}
$$

This "straight" trajectory is uniquely determined since there is no parameter in Eq. (20).

\subsection{Trajectory design}

Here, two straight trajectories are given using Eq. (20) when the constant thrust condition is applied. Calculation conditions are summarized in Tables 1 and 2. Figures 6

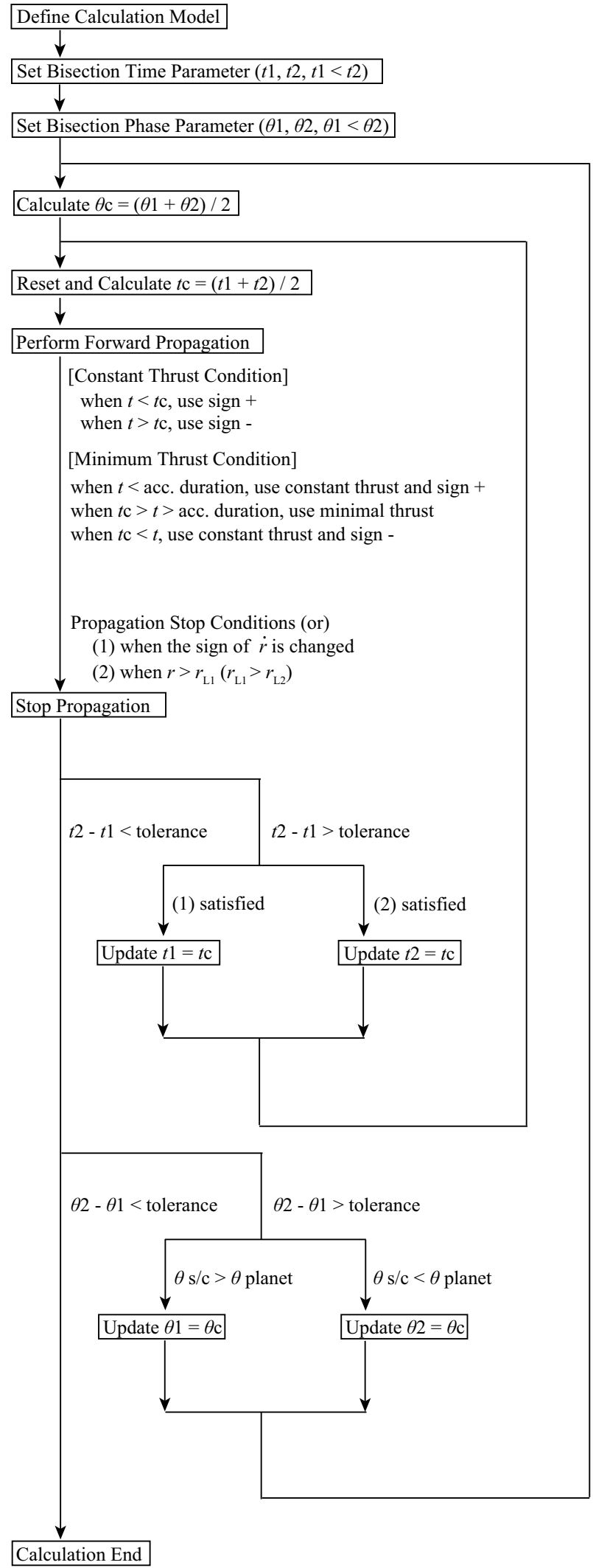

Fig. 5. Flowchart.

and 8 show the designed trajectories: one is Earth-to-Mars transfer and the other is Earth-to-Venus transfer. In these examples, the calculated switching point is the discontinuous point, as clearly shown in Figs. 7 and 9. 
Table 1. Calculation conditions in two-body problems: Case 1.

\begin{tabular}{ll}
\hline Model & $\begin{array}{l}\text { Sun-S/C } \\
\text { two-body problems } \\
\text { (Circular-to-circular transfer) }\end{array}$ \\
& Earth \\
Initial position & Zero w.r.t Earth \\
Initial velocity & Mars \\
Terminal position & Zero w.r.t Mars \\
Terminal velocity & $0.05 \mathrm{~mm} / \mathrm{sec}^{2}$ \\
Acceleration of & \\
the spacecraft (const.) & \\
\hline
\end{tabular}

Table 2. Calculation conditions in two-body problems: Case 2.

\begin{tabular}{ll}
\hline Model & $\begin{array}{l}\text { Sun-S/C } \\
\text { two-body problems } \\
\text { (Circular-to-circular transfer) }\end{array}$ \\
& Earth \\
Initial position & Zero w.r.t Earth \\
Initial velocity & Venus \\
Terminal position & Zero w.r.t Venus \\
Terminal velocity & $0.4 \mathrm{~mm} / \mathrm{sec}^{2}$ \\
Acceleration of & \\
\hline the spacecraft (const.) & \\
\hline
\end{tabular}
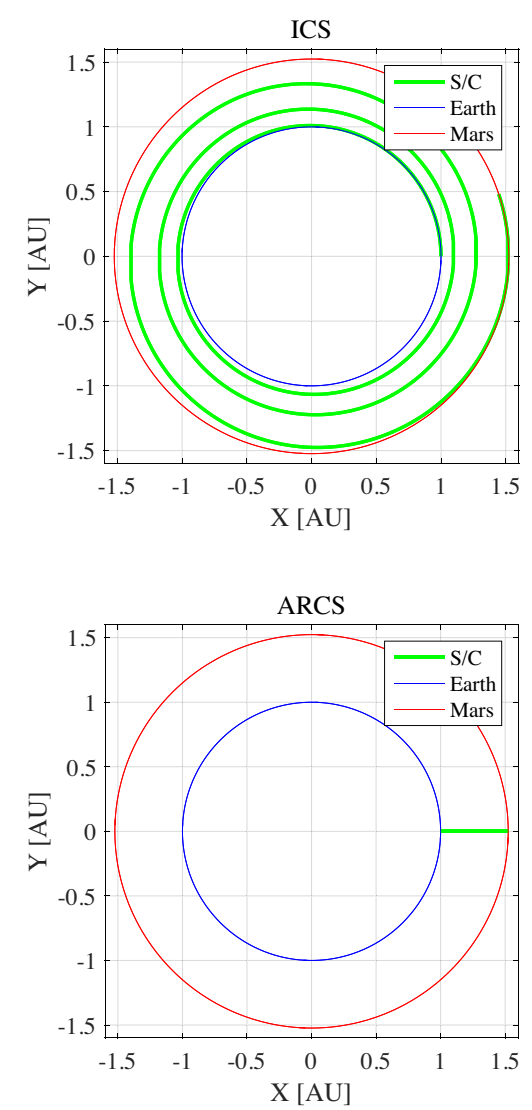

Fig. 6. Straight trajectory: Case 1 (upper: ICS, lower: ARCS).

\subsection{Discussion}

When using the straight trajectory, the tangential velocity of the spacecraft in the ICS and the circular velocity defined in the two-body problems are identical. In a system with a strong gravity field with respect to the propulsion system, such as an Earth-centered system or a Jupiter-centered system, the spiral trajectories have a much larger number of rev-
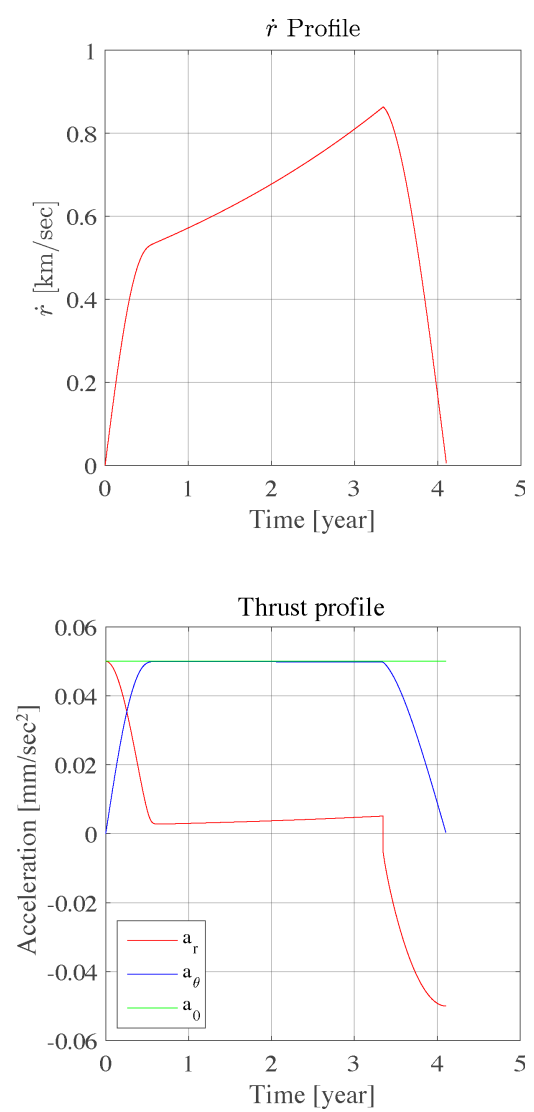

Fig. 7. Trajectory profiles: Case 1 (upper: $\dot{r}$ profile, lower: thrust profiles).
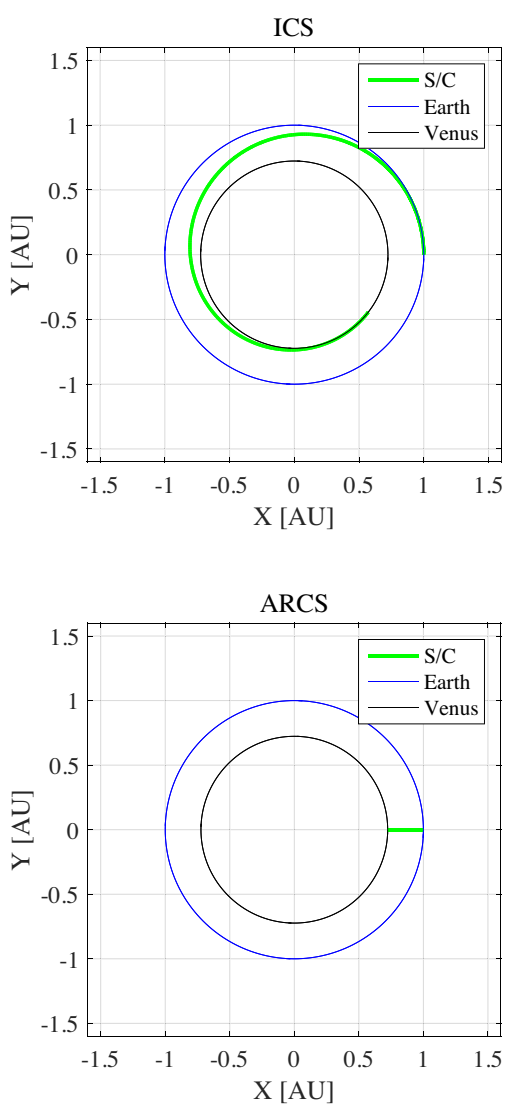

Fig. 8. Straight trajectory: Case 2 (upper: ICS, lower: ARCS). 

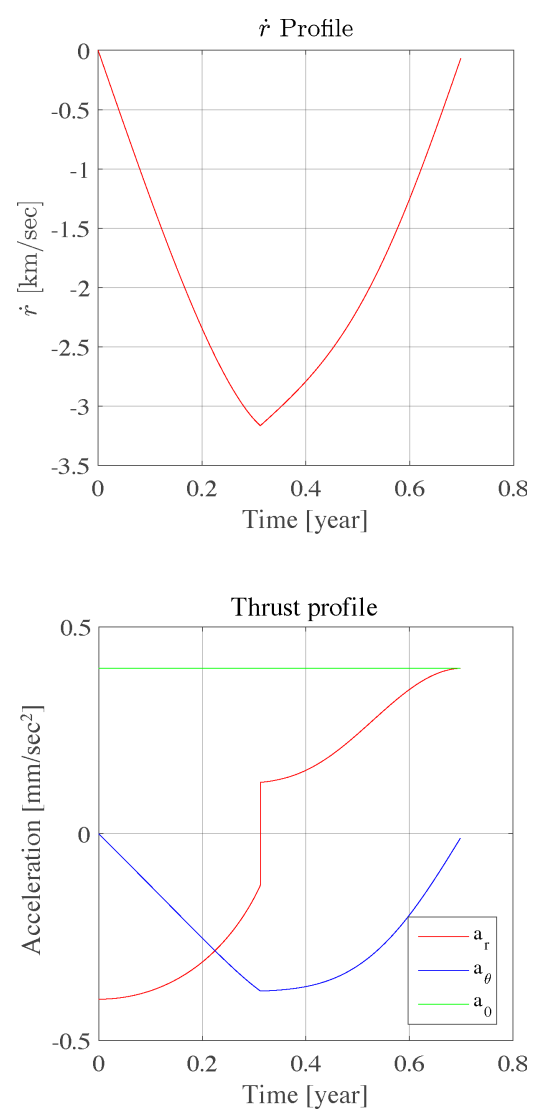

Fig. 9. Trajectory profiles: Case 2 (upper: $\dot{r}$ profile, lower: thrust profiles).

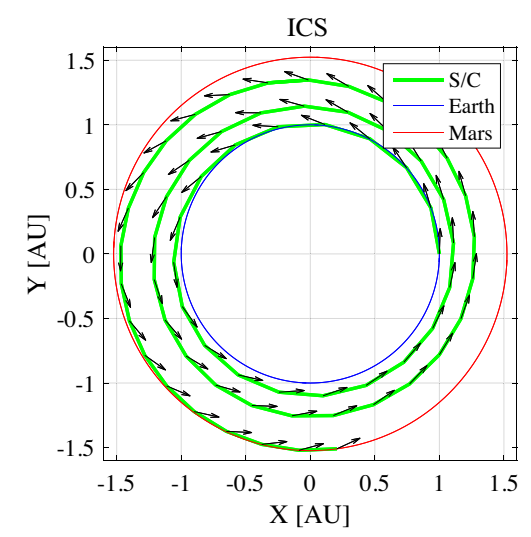

Fig. 10. Optimal trajectory: Case 1 (arrow: thrust vector).

olutions. In this case, the straight trajectory is a good initial guess for the optimal trajectory. Figure 10 shows the minimal flight time trajectory calculated using the DCNLP strategy applying Table 1 calculation conditions. If the straight trajectory shown in Fig. 6 is applied as the initial guess, this optimized calculation is successfully converged. However, if the classical Hohmann transfer trajectory is applied as the initial guess, this optimized calculation is diverged. An advantage of the straight trajectory as the initial guess is that this trajectory satisfies the initial and terminal constraints. This point contributes to increasing the success rate of the optimization calculation.

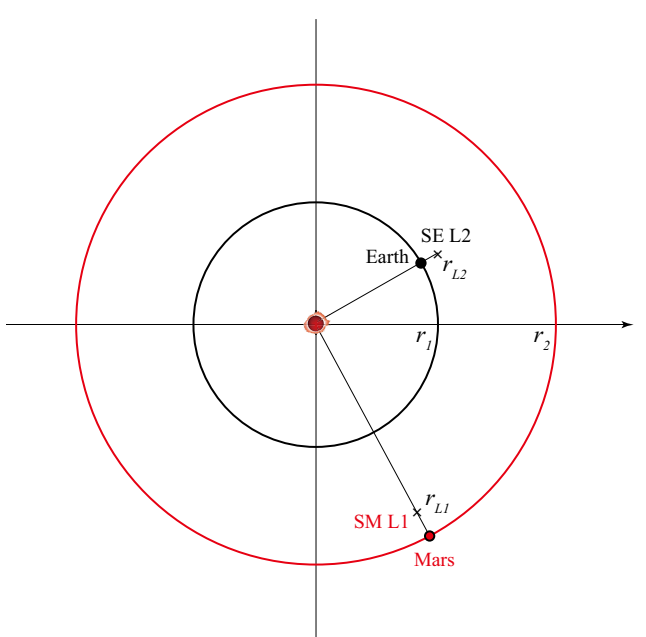

Fig. 11. Circular restricted four-body problems.

\section{Four-Body Problems}

In this section, a more complex trajectory, such as SunEarth L2 to Sun-Mars L1 transfer is designed. Figure 11 shows the model assumed, which is a circular restricted four-body problem. Note that the phase of the planet is considered here.

\subsection{Formulation}

Definition of the ARCS In this problem, the angular velocity of the ARCS is defined by the following equations:

$$
\begin{aligned}
\omega(r) & =\sqrt{\frac{\mu}{r^{* 3}}}, \\
r^{*} & =\frac{r_{\mathrm{L} 1}-r}{r_{\mathrm{L} 1}-r_{\mathrm{L} 2}} r_{1}+\frac{r-r_{\mathrm{L} 2}}{r_{\mathrm{L} 1}-r_{\mathrm{L} 2}} r_{2} .
\end{aligned}
$$

Following this definition, the angular velocity of the ARCS is calculated as:

$$
\begin{aligned}
& \omega\left(r_{\mathrm{L} 2}\right)=\sqrt{\frac{\mu}{r_{1}^{3}}}, \\
& \omega\left(r_{\mathrm{L} 1}\right)=\sqrt{\frac{\mu}{r_{2}^{3}}},
\end{aligned}
$$

when the spacecraft is at Sun-Earth L2 and at Sun-Mars L1, respectively. This means that the velocity of the Lagrange points becomes zero at Sun-Earth L2 and Sun-Mars L1 in the ARCS when the spacecraft is at these points.

Definition of shape function As a shape function, a logistic function defined as

$$
\theta=\phi_{l}(r)=\frac{A}{1+B \exp \{-C(r-D)\}}-\theta_{0}
$$

is applied as an example, where $\theta_{0}$ is a constant value and $r$ is normalized by the astronomical unit (AU). In the actual calculation, without loss of generality, $\theta_{0}$ is set as

$$
\theta_{0}=\frac{A}{1+B \exp \left\{-C\left(r_{\mathrm{L} 2}-D\right)\right\}}
$$




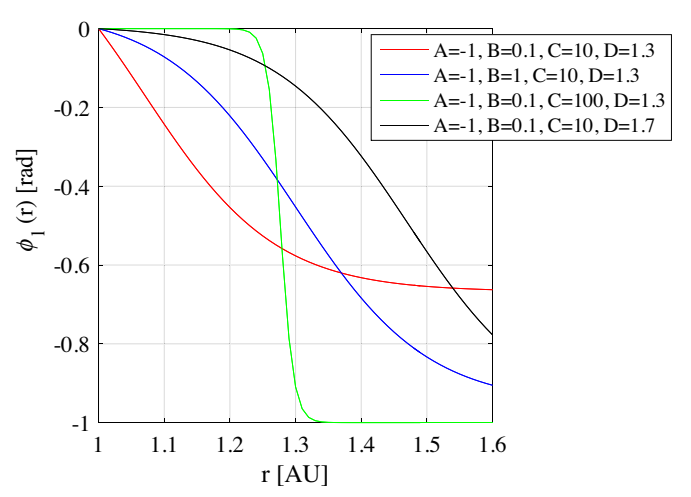

Fig. 12. The shape of the logistic function.

Table 3. Calculation conditions in four-body problems.

\begin{tabular}{ll}
\hline Model & $\begin{array}{l}\text { Sun-Earth-Mars-S/C } \\
\text { circular restricted } \\
\text { four-body problems }\end{array}$ \\
& $\begin{array}{l}\text { Sun-Earth L2 } \\
\text { Zero w.r.t Sun-Earth L2 }\end{array}$ \\
Initial position & Sun-Mars L1 \\
Initial velocity & Zero w.r.t Sun-Mars L1 \\
Terminal position & $0.2 \mathrm{~mm} / \mathrm{sec}^{2}$ \\
Terminal velocity & \\
$\begin{array}{l}\text { Acceleration } \\
\text { of the spacecraft (Max) }\end{array}$ & 0.05 year \\
Acceleration duration & \\
\hline (Min. thrust condition) &
\end{tabular}

Table 4. Calculation parameters.

\begin{tabular}{ll}
\hline Total & 1513 cases \\
$A$ & $0.1,0.01,0.001,0,-0.001,-0.01,-0.1$ \\
$B$ & $0.01,0.1,1,10$ \\
$C$ & $10,20,30,40,50,60,70,80,90$ \\
$D$ & $1.1,1.15,1.2,1.25,1.3,1.35,1.4$ \\
\hline
\end{tabular}

for

$$
\phi_{l}\left(r_{\mathrm{L} 2}\right)=0 .
$$

Figure 12 shows the shape of the logistic function. This function has several parameters, and the shape varies drastically as a function of different selections in the previous parameters. This feature is suitable for designing several kinds of trajectories using a parametric study.

\subsection{Parametric study}

Here, several trajectories that satisfy the boundary conditions are designed by changing the parameters defined in the shape function (Eq. (27)). Tables 3 and 4 show the calculation conditions of this parametric study. In this calculation, the gravitational perturbations and the phases of Earth and Mars are considered.

Constant thrust condition First, the algorithm using constant thrust condition is applied. In this case, 275 trajectories that satisfy the constant thrust condition are generated (others are not physically feasible trajectories). Figure 13 shows the $\Delta V$ vs the time of flight plot. In this configuration, required $\Delta V$ is proportional to the time of flight. Figures 14 and 15 show the trajectory where the required $\Delta V$ is minimal along the trajectories generated.

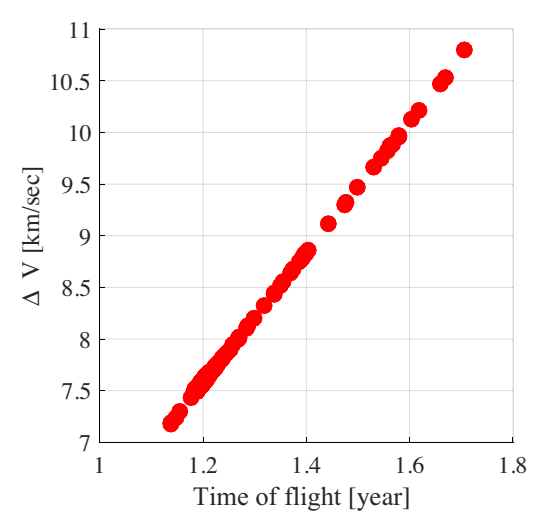

Fig. 13. Trajectories designed (constant thrust condition).
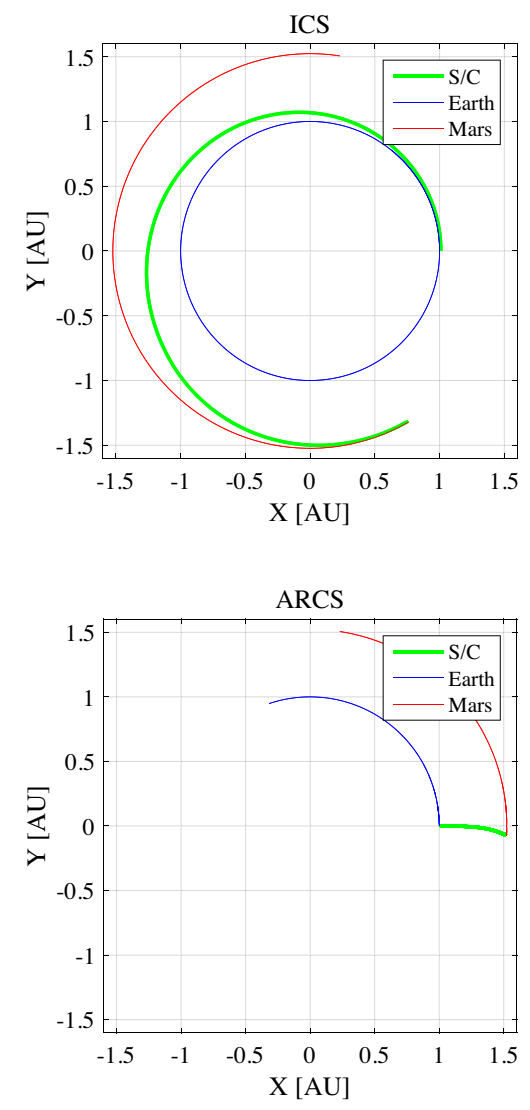

Fig. 14. Trajectory (1) (upper: ICS, lower: ARCS).

Minimum thrust condition Second, the algorithm using the minimal thrust condition is applied. In this case, 782 trajectories that satisfy the minimal thrust condition are generated. Figure 16 shows the $\Delta V$ vs the time of the flight plot, and Figs. 17 and 18 show the trajectory where the required $\Delta V$ is minimum along the trajectories generated.

\subsection{Discussion}

Considering the trajectories generated, the spacecraft usually moves in the $-\theta$ direction in the ARCS due to the Coriolis force. Therefore, the logistic function where parameter $A$ is negative matches the motion induced by the Coriolis force. Figure 19 shows the parameter sensitivity plots for the minimal thrust condition. The upper figure indicates that the flight time is almost the same when parameter $A$, which is 

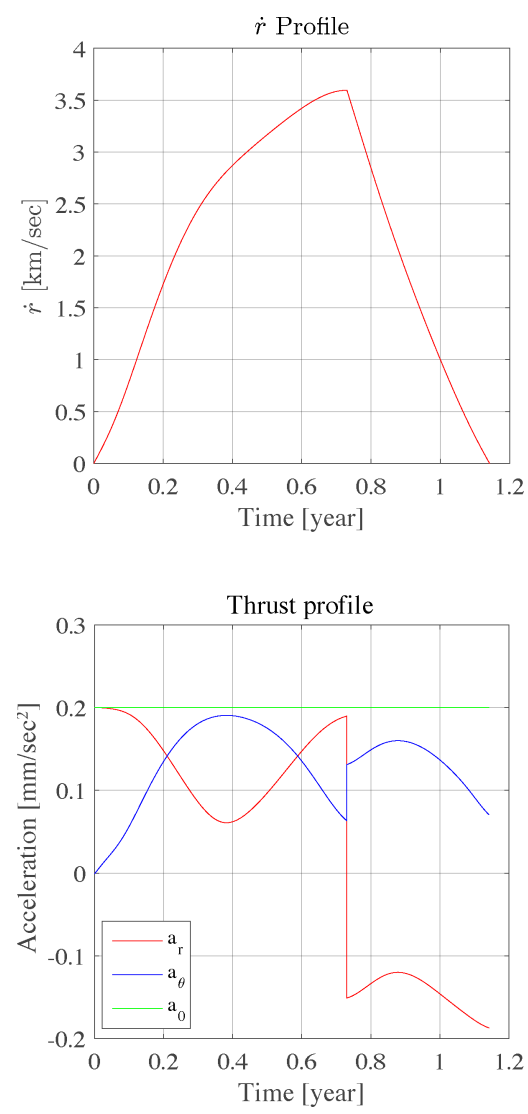

Fig. 15. Trajectory profiles (1) (upper: $\dot{r}$ profile, lower: thrust profiles).

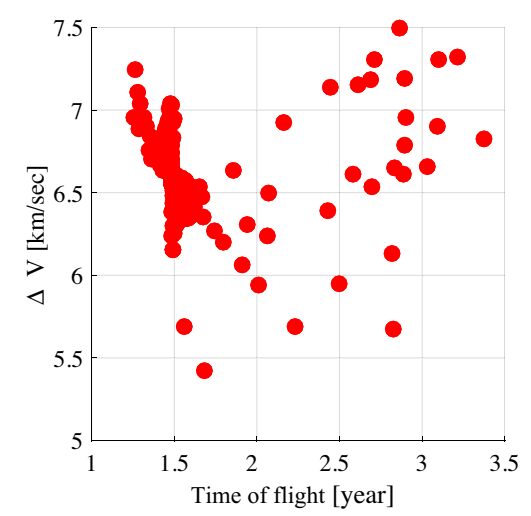

Fig. 16. Trajectories designed (minimal thrusting condition).

larger than -0.01 , is selected. This means that a wide variety of trajectories cannot be designed with large parameter $A$. In addition, the lower figure indicates that the flight time can be selected by changing parameter $C$. According to Fig. 12, parameter $C$ defines the rate of change of the logistic function shape. Therefore, in this case, $A$ and $C$ are key parameters to generate a wide variety of trajectories.

A comparison of Figs. 15 and 18 shows that the minimized thrust condition is more efficient since the acceleration direction and tangential direction are approximately identical. Thus, acceleration toward the $r$ direction is automatically cut off in the minimal thrust condition. This feature contributes to reducing the required $\Delta V$. In addition, many trajectories that have a wide variety of the $\Delta V$ and the time of flight
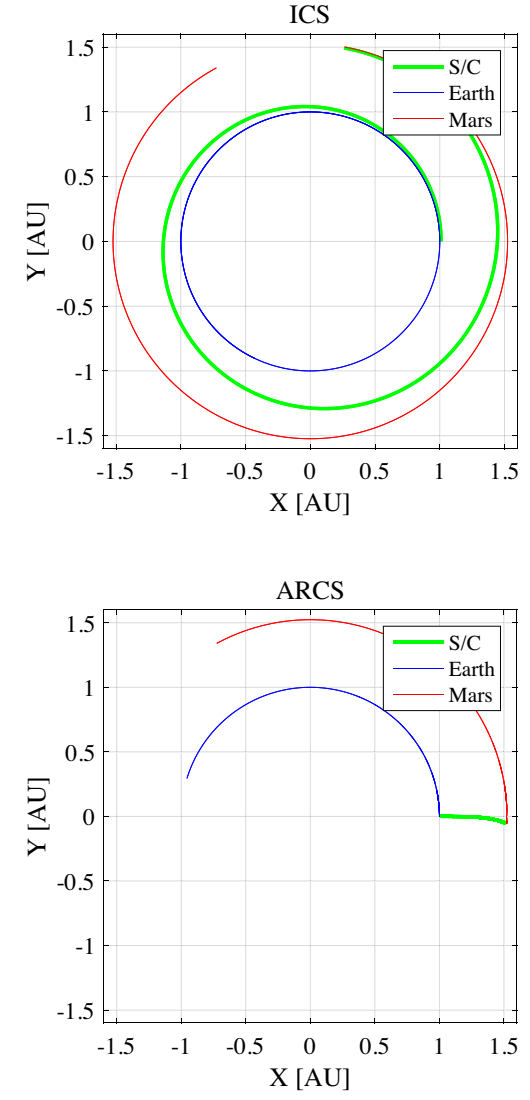

Fig. 17. Trajectory (2) (upper: ICS, lower: ARCS).
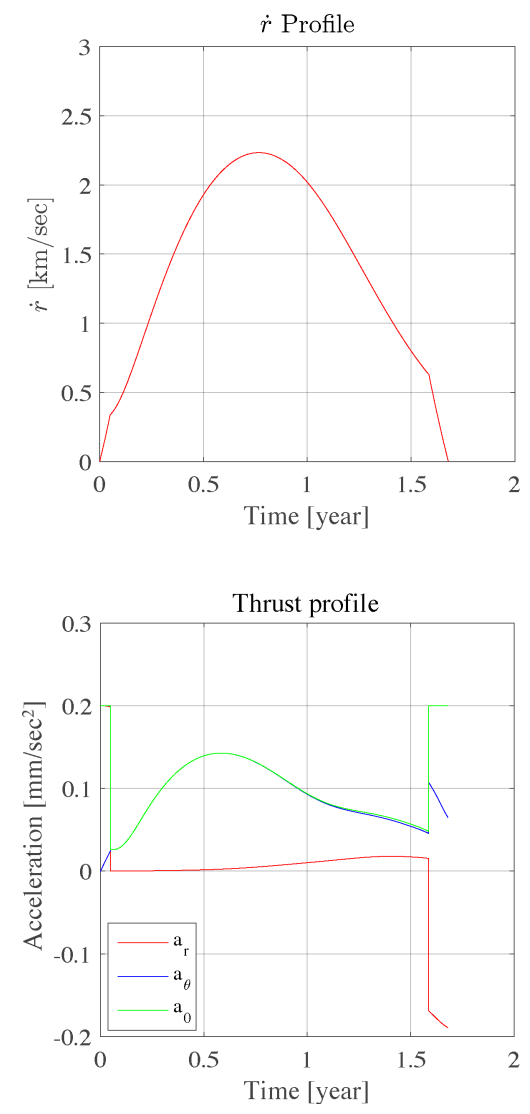

Fig. 18. Trajectory profiles (2) (upper: $\dot{r}$ profile, lower: thrust profiles). 

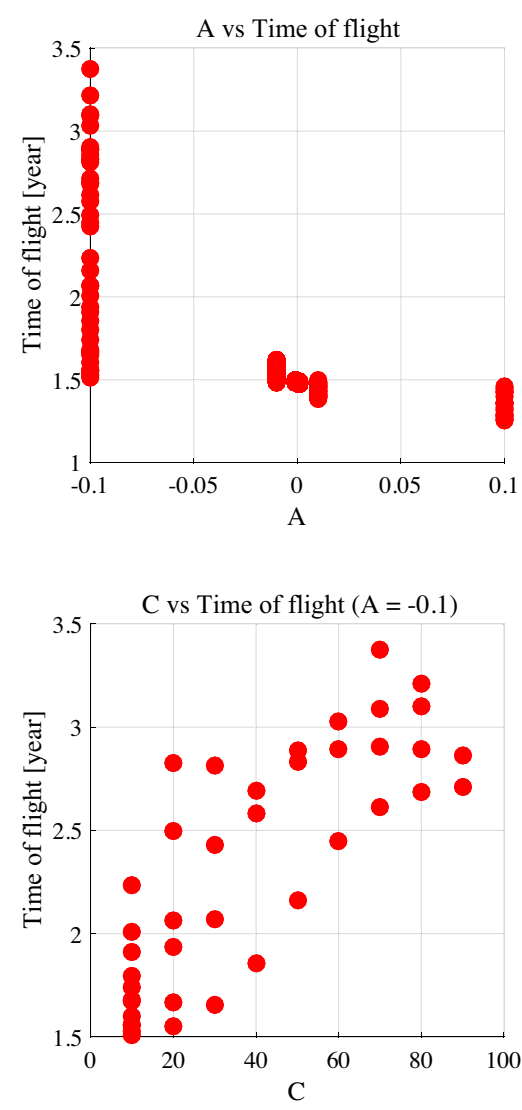

Fig. 19. Sensitivity analysis: minimal thrust condition (upper: $A$ vs time of flight, lower: $C$ vs time of flight).

can be generated during the minimal thrust as shown in Fig. 16. The trajectories generated are usefully applied to the numerical optimal calculation as an initial guess.

\section{Conclusion}

This paper proposed a new description for the spiral trajectory defined in the alternating rotational coordinate system and the shape-based trajectory designing strategy in the ARCS. It was demonstrated that the ARCS has a great advantage of being able to enable the several kinds of shape functions to be designed due to the simplicity in the shape of the spiral trajectories. In addition, the boundary conditions are further simplified if the angular velocity of the ARCS is properly determined. The methodology used in this paper considers several astrodynamical examples of low-thrust trajectory design through the ARCS approach. It was confirmed that the algorithm proposed was successful and that many trajectories which satisfy the boundary conditions are generated. Finally, the trajectories generated by this strategy can be used as an initial guess for numerical optimization calculations.

\section{Acknowledgments}

This work was supported by JSPS KAKENHI Grant Number JP $25 \cdot 6557$.

\section{References}

1) Rayman, M. D., Chadbourne, P. A., Culwell, J. S., and Williams, S. N.: Mission Design for Deep Space 1: A Low-thrust Technology Validation Mission, Acta Astronautica, 45 (1999), pp. 381-388.

2) Kawaguchi, J., Kuninaka, H., Fujiwara, A., and Uesugi, T.: MUSESC, Its Launch and Early Orbit Operations, Acta Astronautica, 59 (2006), pp. 669-678.

3) Racca, G. D., Marini, A., Stagnaro, L., Dooren, J. V., di Napoli, L., Foing, B. H., Lumb, R., Volp, J., Brinkmann, J., Grünagel, R., Estublier, D., Tremolizzo, E., Mckay, M., Camino, O., Schoemaekers, J., Hechler, M., Khan, M., Rathsman, P., Andersson, G., Anflo, K., Berge, S., Bodin, P., Edfors, A., Hussain, A., Kugelberg, J., Larsson, N., Ljung, B., Meijer, L., Mörtsell, A., Nordebäck, T., Persson, S., and Sjöberg, F.: SMART-1 Mission Description and Development Status, Planetary Space Sci., 50 (2002), pp. 1323-1337.

4) Russell, C. T., Capaccioni, F., Coradini, A., De Sanctis, M. C., Feldman, W. C., Jaumann, R., Keller, H. U., McCord, T. B., McFadden, L. A., Mottola, S., Pieters, C. M., Prettyman, T. H., Raymond, C. A., Sykes, M. V., Smith, D. E., and Zuber, M. T.: Dawn Mission to Vesta to and Ceres, Earth Moon Planet, 101 (2007), pp. 65-91.

5) Tsuda, Y., Yoshikawa, M., Abe, M., Minamino, H., and Nakazawa, S.: System Design of the Hayabusa 2 - Asteroid Sample Return Mission to 1999JU3, Acta Astronautica, 91 (2013), pp. 356-362.

6) Campagnola, S., Ozaki, N., Sugimoto, Y., Yam, C. H., Chen, H., Kawabata, Y., Ogura, S., Sarli, B., Kawakatsu, Y., Funase, R., and Nakasuka, S.: Low-thrust Trajectory Design and Operations of Procyon, the First Deep-space Micro-spacecraft, 25th International Symposium on Space Flight Dynamics 2015, Oct. 2015.

7) Conway, B. A.: Spacecraft Trajectory Optimization, Cambridge University Press, New York, 2014, pp. 1-4.

8) Betts, J. T.: Survey of Numerical Method for Trajectory Optimization, J. Guid. Control Dynam., 21 (1998), pp. 193-207.

9) Kechichian, J. A.: Reformulation of Edelbaum's Low-Thrust Transfer Problem Using Optimal Control Theory, J. Guid. Control Dynam., 20 (1997), pp. 988-994.

10) Petropoulos, A. E. and Longuski, J. M.: Shape-Based Algorithm for Automated Design of Low-Thrust, Gravity-Assist Trajectories, J. Spacecraft Rockets, 41 (2004), pp. 787-796.

11) Wall, B. J. and Conway, B. A.: Shape-Based Approach to Low-Thrust Rendezvous Trajectory Design, J. Guid. Control Dynam., 32 (2009), pp. 95-101.

12) Hargraves, C. R. and Paris, S. W.: Direct Trajectory Optimization Using Nonlinear Programming and Collocation, J. Guid. Control Dynam., 10 (1987), pp. 338-342.

13) Rauwolf, G. A. and Coverstone-Carroll, V. L.: Near-Optimal LowThrust Orbit Transfers Generated by a Genetic Algorithm, J. Spacecraft Rockets, 33 (1996), pp. 859-862.

14) Wie, B.: Space Vehicle Dynamics and Control, Second Edition, American Institute of Aeronautics and Astronautics, Virginia, 2008, pp. 221-238.

Katsuhiko Yamada Associate Editor 